\title{
Formation of three-dimensional surface waves on deep-water using elliptic solutions of nonlinear Schrödinger equation
}

\author{
Shahrdad G. Sajjadi ${ }^{\dagger \dagger}$, Stefan C. Mancas ${ }^{\dagger}$ and Frederique Drullion ${ }^{\dagger}$ \\ ${ }^{\dagger}$ Department of Mathematics, ERAU, Florida, U.S.A. \\ ${ }^{\ddagger}$ Trinity College, University of Cambridge, U.K.
}

\begin{abstract}
A review of three-dimensional waves on deep-water is presented. Three forms of three dimensionality, namely oblique, forced and spontaneous type, are identified. An alternative formulation for these three-dimensional waves is given through cubic nonlinear Schrödinger equation. The periodic solutions of the cubic nonlinear Schrödinger equation are found using Weierstrass elliptic $\wp$ functions. It is shown that the classification of solutions depends on the boundary conditions, wavenumber and frequency. For certain parameters, Weierstrass $\wp$ functions are reduced to periodic, hyperbolic or Jacobi elliptic functions. It is demonstrated that some of these solutions do not have any physical significance. An analytical solution of cubic nonlinear Schrödinger equation with wind forcing is also obtained which results in how groups of waves are generated on the surface of deep water in the ocean. In this case the dependency on the energy-transfer parameter, from wind to waves, make either the groups of wave to grow initially and eventually dissipate, or simply decay or grow in time.

PACS numbers: $02.30 . \mathrm{Hq}, 02.30 . \mathrm{Ik}, 02.30 . \mathrm{Gp}$
\end{abstract}

\section{INTRODUCTION}

Finite amplitude water waves have been studied since the pioneering work of G.G. Stokes in 1847 [1]. Over the years very important methods (such as the method of inverse scattering) have been developed for obtaining exact solutions to water wave problems, for instance, analytical solutions to Korteweg-deVries equation for shallow water waves and the cubic nonlinear Schrödinger equation which describes wave envelopes for slow modulation of weakly nonlinear water waves. The mathematical richness in the field of water waves appears surprising at first sight, since the governing differential equation is simply that of Laplace's equation but, of course, since the boundary conditions are nonlinear this gives rise to wealth of problems. It is also remarkable that in a classical field such as that of water waves, new physical phenomena are still being discovered, both theoretically and experimentally, and many open questions still remain unanswered.

For the two-dimensional periodic, irrotational surface waves of permanent form propagating under the influence of gravity on water of infinite or finite depth, such as Stokes waves, much has been discovered over the last few decades. For example, instability of steady finite amplitude waves to long wave two-dimensional disturbances was first postulated by Lighthill [2 by the use of Whitham's variational principle, and an approximate Lagrangian. Zakharov [3] used Hamiltonian methods and showed that weakly nonlinear gravity waves are unstable for modulations longer than a critical wavelength depending upon their waveheight both analytically and numerically for two as well as three-dimensional disturbances. Benjamin \& Feir 4 examined the case of two-dimensional disturbances to weakly nonlinear waves, by adopting standard perturbation methods, found that two-dimensional disturbances, of sufficiently long wavelength, are unstable for which experimental evidence was demonstrated by Benjamin \& Feir [5] and Feir [6]. In a series of experiments reported by Benjamin \& Feir [5] deep-water wave trains of relatively large amplitude were generated at one end of a tank, and were seen traveling many wavelengths. They observed that these wave trains developed conspicuous irregularities when traveled far enough, and they completely disintegrated. The severity of the instability can be seen in Fig. 1 where the fundamental wavelength is $7.2 \mathrm{ft}$ at the water depth of $25 \mathrm{ft}$. Fig. 1 (a) shows the wave train close to the wavemaker, and Fig. 1 (b) shows the same wave train at a distance of $200 \mathrm{ft}$ (28 wavelengths) further along the tank.

Extension to Benjamin-Feir instability for three-dimensional oblique waves on deep water was first studied by Ross \& Sajjadi [7] using the same perturbation method as that of Benjamin \& Feir [4. They discovered a new instability criterion for these waves which reduces to the classical Benjamin-Feir instability when the angle between the waves is zero [8. They also demonstrated that in the limiting case, where the waves are propagating at an angle $\frac{1}{2} \pi$, the instability represents standing waves such as those studied by Penny \& Price 9 .

Longuet-Higgins [10, 11] investigated, by combination of numerical analytical techniques, the stability of finite amplitude water waves to superharmonic and subharmonic two-dimensional disturbances. His work was an extension of results obtained by Zakharov and Benjamin \& Feir to finite amplitude waves for disturbances of shorter wavelength. Longuet-Higgins analysis 10, 11 confirmed Lighthill's prediction that the long wave instability is no longer present when the waves become very steep (that is when the steepness $a k \gtrsim 0.3$ ) and gave values for growth rates that agree well with the observations of Benjamin \& Feir, as well as Benjamin [12] and Lake \& Yuen [13. Longuet-Higgins also 


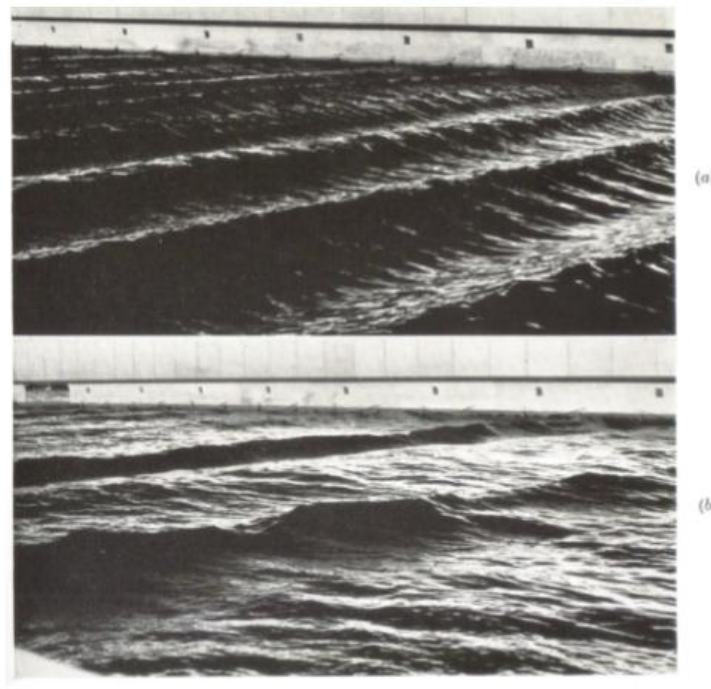

FIG. 1: Photographs of progressive wave trains, of fundamental wavelength $7.2 \mathrm{ft}$, showing disintegration due to Benjamin-Feir instability; (Top) Close to wavemaker; (Bottom) $200 \mathrm{ft}$ away from wavemaker. From Benjamin [12].

discovered that when the wave is sufficiently steep, two-dimensional subharmonic disturbances of twice the wavelength of the undisturbed wave become unstable and have growth rates substantially larger than the type studied by Lighthill, Zakharov and Benjamin \& Feir. Fig. 2 (left) shows such wave patterns up to 23 wavelengths. This photograph shows the first few waves contain small subharmonic disturbances which grow in size and height. These perturbations are three-dimensional, with wavelength of $\frac{1}{3}$ of original basic wave. Fig. 3 (right), on the other hand, depicts the threedimensional spilling breakers due to subharmonic instabilities in which the nature of the crescent-shaped breakers can clearly be seen. As Su et al. [14 commented, the three-dimensionality of the spilling breakers is an intrinsic characteristic of the three-dimensional subharmonic instabilities. 


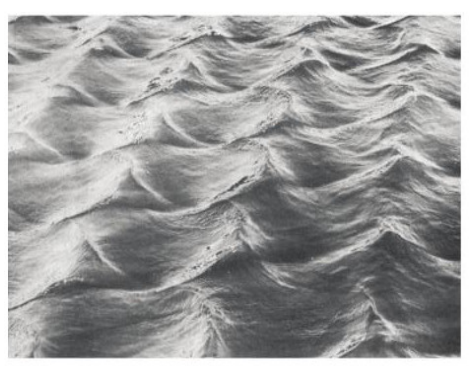

FIG. 2: (Top) Wave patterns in a basin showing two-dimensional subharmonic instabilities $(a k=0.32, f=1.55 \mathrm{~Hz})$. (Bottom) A typical three-dimensional spilling breakers in a basin. From $\mathrm{Su}$ et al. [14].

\section{THREE-DIMENSIONAL WATER WAVES}

Following the advances mentioned above for two-dimensional water waves, attention has been focused to threedimensional propagating water waves, where an even greater richness of phenomena has been encountered [15]. We remark that an important distinction in three-dimensional water waves has to be made, namely between forced and spontaneous three-dimensional waves. The forced three-dimensional water waves is where the dependence upon the second horizontal dimension is forced by boundary or initial conditions. This is the case when one is concerned with studying the effect of nonlinearity on the interaction of two equal but non-parallel wave trains or the reflection of an obliquely incident wave train on a wall [7, see Fig. 3 (bottom). As it has already been remarked, a special case when the waves are at an angle of $\frac{1}{2} \pi$ to each other represents a two-dimensional standing wave 7 .

Forced three-dimensional waves, from a mathematical stand point, are essentially superharmonic modifications of the fundamental waves. The basic linear state is given by 8 ]

$$
\eta(x, y, t)=a \cos [k x \cos \theta+k y \sin \theta-\omega t]+a \cos [k x \cos \theta-k y \sin \theta-\omega t]
$$

with $\omega=\Omega(k)$ being the linear dispersion relation for waves with wavenumber $k$ where two wave trains make an angle $2 \theta$ with each other. The case $\theta=0$ is the limit of two-dimensional propagating waves, and the case $\theta=\frac{1}{2} \pi$ is a twodimensional standing wave. The steady propagating finite amplitude forced three-dimensional waves of permanent form is a solution which may be expressed in the form

$$
\eta(x, y, t)=\sum_{m=0}^{\infty} \sum_{n=0}^{\infty} a_{m n} \cos [m k \cos \theta(x-c t)] \cos [n k \sin \theta y]
$$

where $c$ is the wave complex speed. These wave profiles are commonly referred to as short crested waves and were first studied by Fuchs [16] and Chappelear [17. Perhaps the most important property of these waves is that they exist in the infinitesimal limit, and consequently can be calculated formally by expansions in powers of wave height $h$. However, there are serious concerns as to whether such expansions converge and thus the existence of these steady short crested waves is still not fully determined. For standing waves, that is when $\cos \theta \rightarrow 0$ and with $c$ being finite, the 
first comprehensive study was made by Penney \& Price [9] and later on by Schwartz \& Whitney [18]. The existence of these waves are also uncertain.
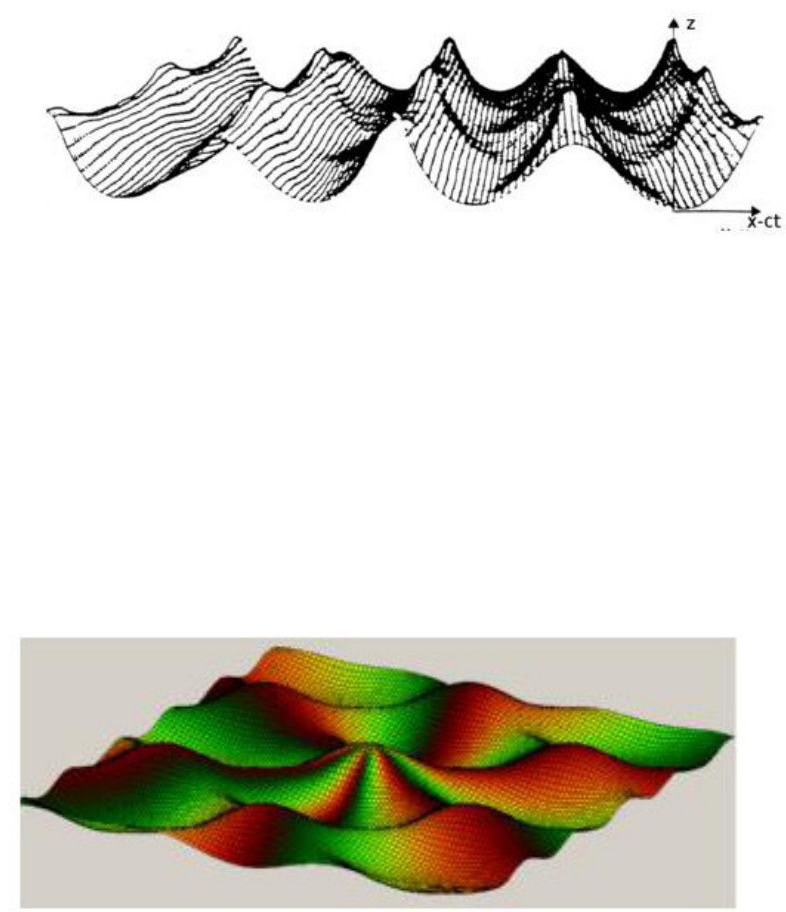

FIG. 3: (Top) A perspective plot of a spontaneous three-dimensional wave for $p=\frac{1}{2}$ and $q=1.2$. (Bottom) A perspective plot of a forced three-dimensional wave for $a k=0.1$.

In contrast, the spontaneous three-dimensional waves are completely different to that of forced three-dimensional waves because these waves originate by instabilities or bifurcation of a uniform two-dimensional wave train and, in general, they cannot have an arbitrary small amplitude. Mathematically speaking, they can be described or interpreted as subharmonic bifurcations [11, where a two-dimensional wave of wavelength $2 \pi / k$

$$
\bar{\eta}(x, t)=\sum_{\ell=0}^{\infty} a_{\ell} \cos \ell k(x-c t)
$$

bifurcates at a critical height into a steadily propagating three-dimensional wave of the form

$$
\eta(x, y, t)=\bar{\eta}(x, t)+\sum_{\ell=0}^{\infty} \sum_{m=-\infty}^{\infty} \sum_{n=-\infty}^{\infty} A_{\ell, m, n} \cos [(\ell+m p) k(x-c t)+k n q y]
$$

where $p$ and $q$ are arbitrary real numbers with $0<p<1$. However, if $p$ is an integer, these represent short crested waves. The critical wave height at which bifurcation occurs depends upon the values of $p$ and $q$. The surface elevation, $\eta(x, y, t)$, given by (2.4), is periodic in the transverse direction and having wavelength $2 \pi / k q$. The longitudinal variation of these waves can be thought of having wavelength $2 \pi / k p$. Note incidentally, these waves are not exactly periodic unless $p$ is rational. In the particular case when $p=1 / 2$ these waves correspond to those whose wavelength is doubled in the direction of propagation. 
The existence of such waves was first demonstrated by Saffman \& Yuen 19 for general nonlinear dispersive systems where the nonlinearity is described by four wave interactions and their detailed calculations for water waves can be found in the paper Saffman \& Yuen [15] using the Zakharov equation. Saffman \& Yuen have pointed out that if the medium is isotropic, the bifurcation is degenerate and the solutions on the new branches can be either skew or symmetric. For skew branches $A_{\ell, m, n} \neq A_{\ell, m,-n}$ and in this case the wave surface is not symmetric about the direction of propagation. On the other hand, for symmetric branches $A_{\ell, m, n}=A_{\ell, m,-n}$ and the surface is symmetric about the direction of propagation as in short crested waves. In this case, the surface elevation may be described by

$$
\eta(x, y, t)=\bar{\eta}(x, t)+\sum_{\ell=0}^{\infty} \sum_{m=-\infty}^{\infty} \sum_{n=0}^{\infty} A_{\ell, m, n}(p, q) \cos [(\ell+m p)(x-c t)] \cos (n q y)
$$

with the corresponding velocity potential, satisfying equations 3.1 below, is given by

$$
\phi(x, y, z, t)=\bar{\phi}(x, t)+\sum_{\ell=0}^{\infty} \sum_{m=-\infty}^{\infty} \sum_{n=0}^{\infty} B_{\ell, m, n}(p, q) \exp \left(\omega_{\ell, m, n} y\right) \sin [(\ell+m p)(x-c t)] \cos (n q y)
$$

where $\omega_{\ell, m, n}=\left[(\ell+m p)^{2}+n^{2} q^{2}\right]$, and both $A_{\ell, m, n}$ and $B_{\ell, m, n}$ are Fourier coefficients, see Section 3. Solutions of this type have been calculated by Meiron et al. [20] using the exact water wave equations [3.1]. A typical example is shown in Fig. 3 (top).

FIG. 4: Three-dimensional symmetric waves $(a k=0.33, f=1.2 \mathrm{~Hz})$ in a wide basin. From $\mathrm{Su}$ [21].

A typical experimental example of symmetric waves in a wide basin generated by a wavemaker with frequency $f=1.2 \mathrm{~Hz}$, wave steepness $a k=0.33$ and wavelength $\lambda=1.08 \mathrm{~m}$ is shown in Fig. 5 . We remark that the antisymmetrical modes of skew waves will lead to a branch of bifurcated solutions in which the surface can be expressed 
as

$$
\eta(x, y, t)=\sum_{m=0}^{\infty} \sum_{n=-\infty}^{\infty} A_{m, n} \cos \left[\frac{1}{2} m(x-c t)+n q y\right]
$$

with $A_{m, n} \neq A_{m,-n}$. These skew waves have the property that a frame of reference can be chosen in which the surface is stationary.
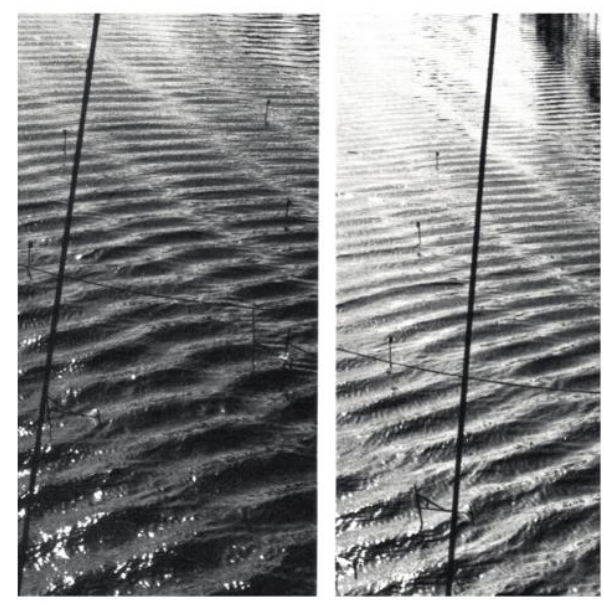

FIG. 5: Skew wave patterns for wave steepness $a k=0.17$. From $\mathrm{Su}$ 14.

Fig. 6, on the other hand, depicts photographs of four skew patterns that propagate to the right side of the $x$-axis. These are three-dimensional wave-forms of the skew wave groups in which each pattern propagates at an angle $\alpha$ with respect to the $x$-direction of the initial Stokes wave train. It is to be noted that skew waves are generally observed in a narrow range of steepness of about $0.16 \leq a k \leq 0.18$. However, the predominantly two-dimensional envelope modulations of Benjamin-Feir instability are uniform over a wider range of steepness $0.1 \leq a k \leq 0.25$ [21, 22]. This observation implies that the bifurcation rate for the skew waves is much lower than the growth rate of Benjamin-Feir instability for $a k<0.16$ and $a k>0.18$ [21].

The approach to the instability and shapes of permanent waves, described above, relies on solving exact formulations, given by equations 3.1. An alternative approach, valid for weak nonlinearity and slow modulations, is solving the cubic nonlinear Schrödinger equation exactly.

The aim of this paper is to classify various possible exact solutions of the elliptic ordinary differential equation that arises from nonlinear Schrödinger equation under the decomposition given by 4.1. To the best of our knowledge 
as yet no such classification has been reported in the open literature. We anticipate the classification presented in this paper will assist in adopting physical solutions to nonlinear Schrödinger equation, for three-dimensional waves, amongst various possible ones.

\section{FORMULATION OF THE PROBLEM}

The governing equations for an irrotational, inviscid, incompressible surface gravity waves on deep water are given by

$$
\begin{aligned}
& \nabla^{2} \phi=0, \quad-\infty<z<\eta(x, y, t) \\
& \left.\begin{array}{l}
\frac{\partial \phi}{\partial t}+\frac{1}{2}|\nabla \phi|^{2}+g \eta=0, \\
\frac{\partial \eta}{\partial t}+(\boldsymbol{\nabla} \eta \cdot \nabla \phi)-\frac{\partial \phi}{\partial z}=0,
\end{array}\right\} \quad z=\eta(x, y, t) \\
& \nabla \phi \rightarrow 0 \quad z \rightarrow-\infty
\end{aligned}
$$

where $g$ is the acceleration due to gravity, $\phi(x, y, z, t)$ is the velocity potential, and $\eta(x, y, t)$ describes the free surface elevation.

Following [3] the surface elevation $\eta(\boldsymbol{x}, t)$, where $\boldsymbol{x}=(x, y)$, of weakly nonlinear deep-water gravity waves may be expressed as

$$
\eta(\boldsymbol{x}, t)=\frac{1}{2 \pi} \int_{-\infty}^{\infty} \frac{|\boldsymbol{k}|^{\frac{1}{4}}}{2^{\frac{1}{2}} g^{\frac{1}{4}}}\left[B(\boldsymbol{k}, t) \mathrm{e}^{i(\boldsymbol{k} \cdot \boldsymbol{x}-\omega t)}+B^{*}(\boldsymbol{k}, t) \mathrm{e}^{-i(\boldsymbol{k} \cdot \boldsymbol{x}-\omega t)}\right] \mathrm{d} \boldsymbol{k}
$$

where superscript $*$ denotes complex conjugates and $\omega$ is the wave frequency which satisfies the dispersion relation

$$
\omega(\boldsymbol{k})=\sqrt{g|\boldsymbol{k}|}
$$

In equation (3.2) $B(\boldsymbol{k}, t)$ is the time evolution of spectral components of a weakly nonlinear system for dominating four-wave interactions and its governing equation is given by

$$
\begin{array}{r}
i \frac{\partial B(\boldsymbol{k}, t)}{\partial t}=\iint_{-\infty}^{\infty} \int T\left(\boldsymbol{k}, \boldsymbol{k}_{1}, \boldsymbol{k}_{2}, \boldsymbol{k}_{3}\right) \delta\left(\boldsymbol{k}+\boldsymbol{k}_{1}-\boldsymbol{k}_{2}-\boldsymbol{k}_{3}\right) \exp \left\{i\left[\omega(\boldsymbol{k})+\omega\left(\boldsymbol{k}_{1}\right)-\omega\left(\boldsymbol{k}_{2}\right)-\omega\left(\boldsymbol{k}_{3}\right)\right] t\right\} \\
\times B^{*}\left(\boldsymbol{k}_{1}, t\right) B\left(\boldsymbol{k}_{2}, t\right) B\left(\boldsymbol{k}_{3}, t\right) \mathrm{d}^{3} k_{1} \mathrm{~d}^{3} k_{2} \mathrm{~d}^{3} k_{3}
\end{array}
$$

where $\omega(\boldsymbol{k})$ is the linear frequency and the real interaction coefficient $T\left(\boldsymbol{k}, \boldsymbol{k}_{1}, \boldsymbol{k}_{2}, \boldsymbol{k}_{3}\right)$, given by originally by Zakharov [3] and later, with some minor corrections, by Crawford et al. [23], characterize the properties of the system. For clarity we have listed these coefficients in the Appendix.

Zakharov [3] in his pioneering paper showed that equations (3.2)- 3.4 yield the surface elevation for deep-water gravity wave (note, in his original paper he also took into account the surface tension) may be reduced to

$$
\eta(\boldsymbol{x}, t)=\operatorname{Re}\left\{A(\boldsymbol{x}, t) \mathrm{e}^{i\left(k_{0}-\omega_{0} t\right)}\right\}
$$

where $A(\boldsymbol{x}, t)=a(\boldsymbol{x}, t) \mathrm{e}^{i \theta(\boldsymbol{x}, t)}$ is the complex envelope of the slowly modulated carrier wave propagating in the $\boldsymbol{x}$-direction. The real envelope is given by $a(\boldsymbol{x}, t)$, and $\boldsymbol{\nabla}_{x} \theta$ and $\partial \theta / \partial t$ represent, respectively, the modulation wave vector and frequency. Zakharov showed that $A(\boldsymbol{x}, t)$ satisfies the two-dimensional nonlinear Schrödinger equation

$$
i\left(\frac{\partial A}{\partial t}+\frac{\omega_{0}}{2 k_{0}} \frac{\partial A}{\partial x}\right)-\frac{\omega_{0}}{8 k_{0}^{2}} \frac{\partial^{2} A}{\partial x^{2}}+\frac{\omega_{0}}{4 k_{0}^{2}} \frac{\partial^{2} A}{\partial y^{2}}-\frac{1}{2} \omega_{0} k_{0}^{2}|A|^{2} A=0
$$

Note incidentally, the complex envelope function $A(\boldsymbol{x}, t)$ is related to the Fourier components $B(\boldsymbol{k}, \tau)$ through the equation

$$
A(\boldsymbol{x}, t)=\frac{\varepsilon}{\pi} \frac{k_{0}}{2 \omega_{0}} \int_{-\infty}^{\infty} B(\boldsymbol{k}, t) \exp \left\{i\left(\boldsymbol{k}-\boldsymbol{k}_{0}\right) \cdot \boldsymbol{x}-i\left[\omega(\boldsymbol{k})-\omega_{0}\right] t\right\} \mathrm{d} \boldsymbol{k}
$$


Zakharov [3] also showed that for oblique plane modulations, where $\xi=x \cos \alpha+y \sin \alpha$, equation (3.6) may be reduced to one-dimensional nonlinear schrödinger equation

$$
i\left(\frac{\partial A}{\partial t}+\frac{\omega_{0}}{2 k_{0}} \cos \alpha \frac{\partial A}{\partial \xi}\right)-\frac{\omega_{0}}{8 k_{0}^{2}}\left(1-\sin ^{2} \alpha\right) \frac{\partial^{2} A}{\partial \xi^{2}}-\frac{1}{2} \omega_{0} k_{0}^{2}|A|^{2} A=0
$$

As was originally shown by Saffman \& Yuen [24] for $\alpha<\sin ^{-1}(1 / 3)=19.47^{\circ}$, equation 3.7 has the following soliton solution

$$
A(\xi, t)=a_{0} \operatorname{sech}\left\{\frac{k_{0}^{2} a_{0}}{\sqrt{1-3 \sin \alpha}}\left(\xi-\frac{\omega_{0}}{2 k_{0}} t \cos \alpha\right)\right\} \exp \left(-\frac{1}{4} i \omega_{0} k_{0}^{2} a_{0}^{2} t\right)
$$

whose profile is depicted in Fig. 6 for $\alpha=\frac{1}{12} \pi$. We remark that for $\alpha>19.47^{\circ}$, there are no steady solutions to equation (3.7) that decay as $|\xi| \rightarrow \infty$. Note that Saffman \& Yuen [24] state that no steady solution exist for $\alpha>35.26^{\circ}$ and they plot their result, using equation (3.8), for the angle $\alpha=30^{\circ}$. This is an error because for $\alpha>19.47^{\circ}$ the term $1-3 \sin \alpha$ will be negative and thus $A(\xi, t)$ will no longer be a sech profile but instead it will yield a periodic solution. Note, in this case $\operatorname{sech}(i \chi)=\sec (\chi)$, where $\chi$ represents the argument of sech in equation (3.8). Fig. 6 (bottom) shows the plot of sec solution for values of arguement slightly below and above the range $-\frac{1}{2} \pi<\chi<\frac{1}{2} \pi$. This is because the sec solution of the equation equation 3.8 becomes unbounded for $\chi=\frac{1}{2}(2 n+1) \pi$ since at these values $\sec (\chi)$ is infinite.

Following a common practice, we adopt the dimensionless variables

$$
T=-\omega_{0} t, \quad X=2 k_{0}\left(x-\frac{\omega_{0}}{2 k_{0}} t\right), \quad Y=2 k_{0} y, \quad \psi=k_{0} A / \sqrt{2}
$$

and obtain the equation 3.6 in non-dimensional form

$$
i\left(\psi_{T}+\frac{1}{2} \psi_{X}\right)-\frac{1}{8} \psi_{X X}+\frac{1}{4} \psi_{Y Y}=\frac{1}{2}|\psi|^{2} \psi
$$

This equation has been used to study stabilities and bifurcation of three-dimensional water waves. This twodimensional form of nonlinear Schrödinger equation (3.9) has attracted a great deal of attention because it can be solved exactly by inverse scattering and other techniques, but we emphasize that this equation has a rather physically limited range of validity. In the range of its validity, however, it provides an easy way to produce all kinds of three-dimensional wave patterns. For example, substituting

$$
\psi(X, Y, T)=f(Y) e^{i(p X-c T)}
$$

into equation 3.9 we obtain

$$
f_{Y Y}+\frac{1}{2} p^{2} f-2 f^{3}+2(2 c-p) f=0 .
$$

Now, depending on the values of the parameters $p$ and $c$, and the magnitude of $f$, many bounded solutions of this equation exist (see Roberts \& Peregrine [25] and Sections 4 and 5 below). For example, there are stationary dislocation-type slip line solutions

$$
f=a \tanh (\sqrt{2} c Y), \quad p=-a^{2},
$$

which describes a surface in which the propagating wave has a phase jump of $\pi$ across the line $Y=0$ (see the case (2a)(ii) below). The problem with the nonlinear Schrödinger equation is that it has an overabundance of solutions, and it is not easy to decide which, if any, are of physical significance. There is also the mathematical problem of determining if the nonlinear Schrödinger solutions are genuine in the sense that there are limits, as the wavenumber of the modulation and the amplitude go to zero, of solutions of the exact equations, and not the leading terms of expansions whose radius of convergence is zero.

\section{ANALYTICAL SOLUTIONS}

To find analytical solutions to 3.9 we employ the polar form of ansatz

$$
\psi(X, Y, T)=f(Y) e^{i \theta}
$$



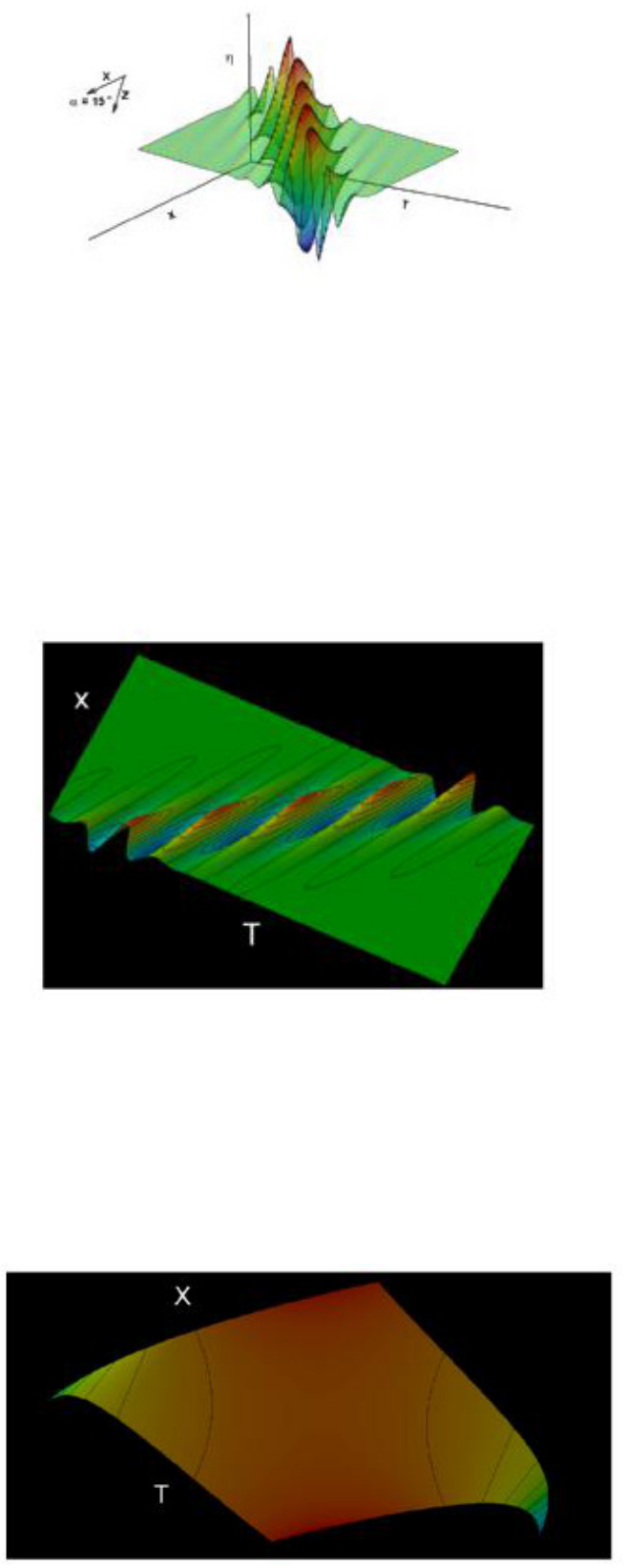

FIG. 6: (Top) Free surface elevation for a plane oblique solitary wave making an angle of $\frac{1}{12} \pi$ with the carrier wave vector. (Middle) A different prospective of three surface elevation as that of the top figure except for an angle $\frac{1}{15} \pi$. (Bottom) is the same as the middle profile for an angle $\frac{1}{6} \pi$, where the sech-profile becomes a sec profile. Note at values $\frac{1}{2}(2 n+1) \pi$, where $n$ is an integer (including zero), the sec-profile becomes infinite. 
with phase $\theta=k X-\omega T, k$ is the wave number, $\omega$ is the angular frequency, and $f(Y)$ is the real magnitude of the field. Substituting the ansatz into $(3.9)$, yields

$$
f_{Y Y}+4 w(k) f=2 f^{3} .
$$

By multiplying by $f_{Y}$ and integrating once we obtain the elliptic equation

$$
f_{Y}^{2}=f^{4}-4 w(k) f^{2}+\mathscr{A}
$$

where $\mathscr{A}$ is the integration constant that depends on the boundary conditions used, and

$$
w(k)=\omega-\frac{k}{2}+\frac{k^{2}}{8}
$$

is the dispersion relation. We will make use of this disperion relation in order to classify the solutions and will be written as $\omega-\omega_{0}$ therein where $\omega_{0}=\frac{1}{2} k-\frac{1}{8} k^{2}$, see Fig. 7 which gives the graph of the dispersion relation.

It is well known [26, 27, that the solutions $f(Y)$ of

$$
f_{Y}^{2}=p_{4}(f)
$$

where $p_{4}(f)$ is a quartic polynomial in $f(Y)$ can be expressed in terms of in Weierstrass elliptic functions $\wp\left(Y ; g_{2}, g_{3}\right)$ via

$$
f(Y)=f_{0}+\frac{\sqrt{p_{4}\left(f_{0}\right)} \wp^{\prime}(z)+\frac{1}{2} p_{4}{ }^{\prime}\left(f_{0}\right)\left(\wp(Y)-\frac{1}{24} p_{4}{ }^{\prime \prime}\left(f_{0}\right)\right)+\frac{1}{24} p_{4}\left(f_{0}\right) p_{4}{ }^{(3)}\left(f_{0}\right)}{2\left(\wp(Y)-\frac{1}{24} p_{4}{ }^{\prime \prime}\left(f_{0}\right)\right)^{2}-\frac{1}{48} p_{4}\left(f_{0}\right) p_{4}^{(4)}\left(f_{0}\right)},
$$

where $f_{0}$ is not necessarily a root of $p_{4}(f)$, and $g_{2}, g_{3}$ are elliptic invariants of $\wp(Y)$. Due to the biquadratic nature of $Q(f)$, an analysis of the nature of solutions of (4.6) is made, and we will show that solutions of (4.6) are reduced to solitary waves, periodic or Jacobi type of elliptic functions.

To see this we let $f^{2}(Y)=\zeta(Y)$ in $(4.3)$ which yields the Weierstrass equation

$$
\zeta_{Y}^{2}=4 \zeta^{3}-16 w(k) \zeta^{2}+4 \mathscr{A} \zeta
$$

Using a linear transformation $\zeta=\hat{\zeta}+\frac{4}{3} w(k)$, 4.7) can be written in normal form

$$
\hat{\zeta}_{Y}^{2}=4 \hat{\zeta}^{3}-g_{2} \hat{\zeta}-g_{3}=4\left(\hat{\zeta}-e_{1}\right)\left(\hat{\zeta}-e_{2}\right)\left(\hat{\zeta}-e_{3}\right)
$$

The germs of the Weierstrass equation 4.8 are

$$
\left.\begin{array}{l}
g_{2}=4\left(\frac{16}{3} \omega^{2}(k)-\mathscr{A}\right)=2\left(e_{1}^{2}+e_{2}^{2}+e_{3}^{2}\right) \\
g_{3}=\frac{16 \omega(k)}{3}\left(\frac{32}{9} \omega^{2}(k)-\mathscr{A}\right)=4\left(e_{1} e_{2} e_{3}\right)
\end{array}\right\},
$$

and together with the modular discriminant

$$
\Delta=g_{2}^{3}-27 g_{3}^{2}=16\left(e_{1}-e_{2}\right)^{2}\left(e_{1}-e_{3}\right)^{2}\left(e_{2}-e_{3}\right)^{2}
$$

are used to classify the solutions of 4.7). Also, $e_{i}$ are the three solutions to the cubic polynomial equation

$$
p_{3}(s)=4 s^{3}-g_{2} s-g_{3}=0,
$$

and are related to the two periods $\omega_{1,2}$ of the $\wp$ function for $e_{i}=\wp\left(\omega_{i} / 2\right)$, and $\omega_{3}=-\left(\omega_{1}+\omega_{2}\right)$, see[28].

Since the solution of equation 4.8 is

$$
\hat{\zeta}=\wp\left(Y ; g_{2}, g_{3}\right)
$$

then the solution of equation (4.3) may be expressed as

$$
f(Y)= \pm \sqrt{\wp\left(Y ; g_{2}, g_{3}\right)+\frac{4 \omega(k)}{3}}
$$




\section{RESULTS}

We shall now proceed to classify the solutions of equation 4.3 case-by-case.

Case (1). We consider the simpler case with zero boundary conditions, that is when $\mathscr{A} \equiv 0$. In this case equations 4.9 become

$$
g_{2}=\frac{2^{6}}{3} \omega^{2}(k) \quad \text { and } \quad g_{3}=\frac{2^{9}}{3^{3}} \omega^{3}(k)
$$

Also, in this case, 4.10 reduces to

$$
\Delta \equiv 0
$$

which implies that $p_{3}(s)$ either has repeated root $e_{i}$ of multiplicity two $(m=2)$ or three $(m=3)$.

Case (1a). $(m=2)$, we now let $e_{1}=e_{2}=u>0$ then $e_{3}=-2 u<0$, hence

$$
g_{2}=12 u^{2}>0 \quad \text { and } \quad g_{3}=-8 u^{3}<0
$$

Also, from equations 4.9 we see that

$$
u=-\frac{4}{3} \omega(k)>0 \quad \text { then } \quad \omega(k)<0 \quad \text { which gives } \omega<\omega_{0}=\frac{k}{2}-\frac{k^{2}}{8},
$$

see Fig. 7 (left), and hence, in this case [28, solution of 4.8 is given by

$$
\wp_{1 a}\left(Y ; 12 u^{2},-8 u^{3}\right)=u+3 u \operatorname{csch}^{2}(\sqrt{3 u} Y) .
$$

Using (5.2, 5.5 in 4.13, we may express the solution of equation 4.3 as

$$
f_{1 a}(Y)=2 \sqrt{-\omega(k)}|\operatorname{csch}(2 \sqrt{-\omega(k)} Y)|
$$

For $k=1, \omega=-1 \rightarrow \omega(k)=-\frac{11}{8}$, equation (5.6) becomes

$$
f_{1 a}(Y)=\frac{\sqrt{22}}{2}\left|\operatorname{csch}\left(\frac{\sqrt{22}}{2} Y\right)\right|
$$

see Fig. 8 (blue).

Case (1b). $(m=2)$, here we let $e_{2}=e_{3}=-u<0$ then $e_{1}=2 u>0$, hence

$$
g_{2}=12 u^{2}>0 \quad \text { and } \quad g_{3}=8 u^{3}>0
$$

Also, from equations 4.9 we have

$$
u=\frac{4}{3} \omega(k)>0 \quad \text { then } \omega(k)>0 \quad \text { which gives } \omega>\omega_{0}=\frac{k}{2}-\frac{k^{2}}{8},
$$

see Fig. 7 (center), thus in this case [28] solution of equation 4.8 is given by

$$
\wp_{1 b}\left(Y ; 12 u^{2}, 8 u^{3}\right)=-u+3 u \csc ^{2}(\sqrt{3 u} Y) .
$$

Using (5.9), 5.10) in 4.13), we may write the solution of 4.3 in the form

$$
f_{1 b}(Y)=2 \sqrt{\omega(k)}|\csc (2 \sqrt{\omega(k)} Y)|
$$

For $k=1, \omega=1$ then $\omega(k)=\frac{5}{8}$, equation (5.11 becomes

$$
f_{1 b}(Y)=\frac{\sqrt{10}}{2}\left|\csc \left(\frac{\sqrt{10}}{2} Y\right)\right|
$$

see Fig. 8(red). 

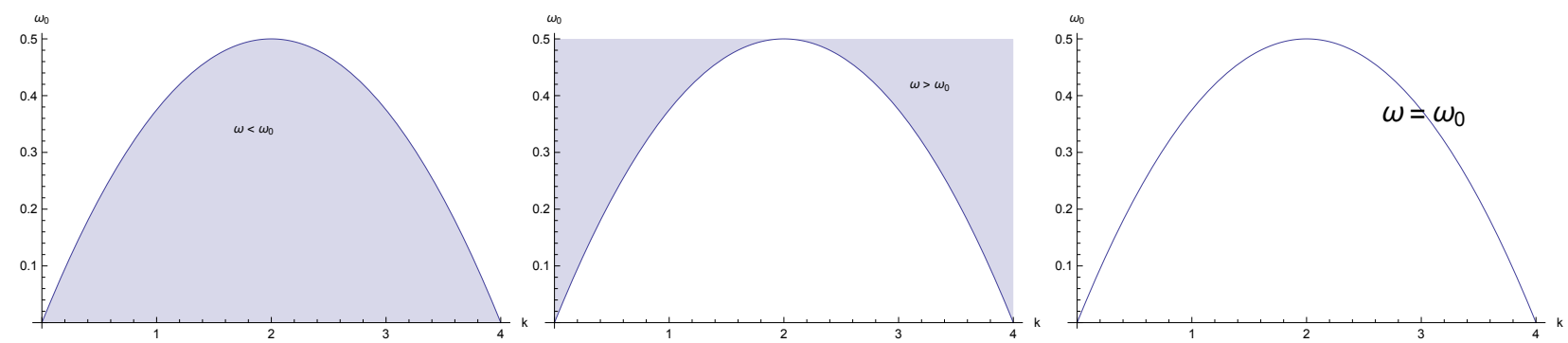

FIG. 7: Domain of solutions $f_{1 a}$ (left), $f_{1 b}$ (center), $f_{1 c}$ (right), when $\mathscr{A}=0$.

Case (1c). $(m=3)$ : here we obtain $e_{1}=e_{2}=e_{3}=0$, hence $g_{2}=g_{3}=0$ then $\omega(k)=0$ and this yields $\omega=\omega_{0}=\frac{k}{2}-\frac{k^{2}}{8}$. For $k=1, \omega=\frac{3}{8}$ then $\omega(k)=0$. Hence

$$
\wp_{1 c}(Y ; 0,0)=\frac{1}{Y^{2}}
$$

and

$$
f_{1 c}(Y)=\frac{1}{Y}
$$

see Fig. 8 (green).

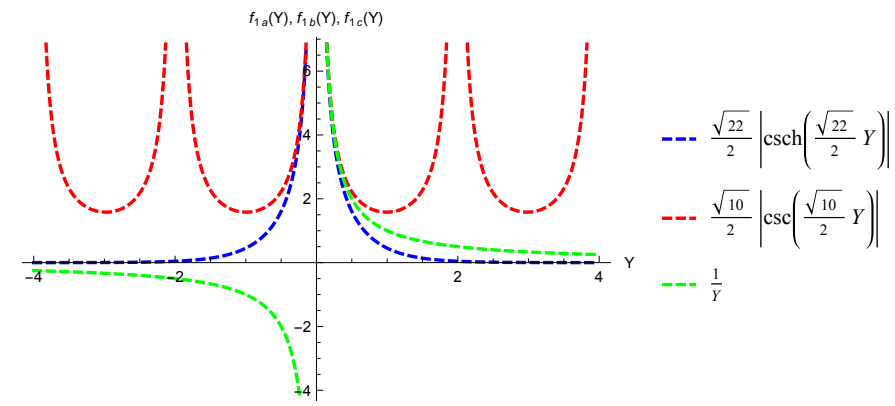

FIG. 8: Solutions when $\mathscr{A}=0$, for $k=1, \omega=-1$ (blue); $k=1, \omega=1$ (red); and $k=1, \omega=\frac{1}{3}$ (green).

Case (2). Now we consider the general case with nonzero boundary conditions, i.e., $\mathscr{A} \neq 0$, then equation 4.3 is factored as

$$
f_{Y}^{2}=\left(f^{2}-a\right)\left(f^{2}-b\right)
$$

where

$$
a, b=2 \omega(k) \pm \sqrt{4 \omega^{2}(k)-\mathscr{A}}
$$

For real solutions of the amplitude $f$, we require $\mathscr{A} \leq 4 \omega^{2}(k)$.

Case (2a). For $\mathscr{A}=4 \omega^{2}(k) \neq 0$ then $a=b=2 \omega(k)$, and equation 5.15 becomes

$$
f_{Y}= \pm\left(f^{2}-a\right)
$$


Case (2a)(i). $\omega(k)<0$, then

$$
f_{2 a i}(Y)=\sqrt{-2 \omega(k)} \tan (\sqrt{-2 \omega(k)} Y) .
$$

Now, for $k=1, \omega=-1$ then $\omega(k)=-\frac{11}{8}$ gives $\mathscr{A}=\frac{121}{16}$, and equation (5.18) becomes

$$
f_{2 a i}(Y)=\frac{\sqrt{11}}{2} \tan \left(\frac{\sqrt{11}}{2} Y\right)
$$

see Fig. 9 (blue).

Case (2a)(ii). $\omega(k)>0$ then

$$
f_{2 a i i}(Y)=\sqrt{2 \omega(k)} \tanh (\sqrt{2 \omega(k)} Y) .
$$

For $k=1, \omega=1 \rightarrow \omega(k)=\frac{5}{8} \rightarrow \mathscr{A}=\frac{25}{16}$ and equation 5.20 becomes

$$
f_{2 a i i}(Y)=\frac{\sqrt{5}}{2} \tanh \left(\frac{\sqrt{5}}{2} Y\right)
$$

see Fig. 9 (red).

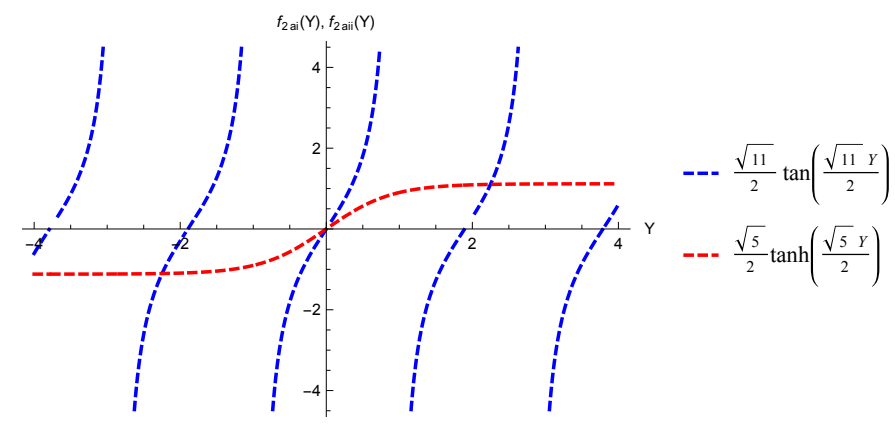

FIG. 9: Solutions when $\mathscr{A}=4 \omega^{2}(k)$, for $k=1, \omega=-1, \mathscr{A}=\frac{121}{16}$ (blue); and $k=1, \omega=1$ (red), $\mathscr{A}=\frac{25}{16}$.

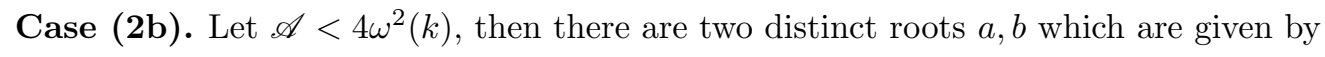

$$
a=2 \omega(k)+\sqrt{4 \omega^{2}(k)-\mathscr{A}} \quad \text { and } \quad b=2 \omega(k)-\sqrt{4 \omega^{2}(k)-\mathscr{A}}
$$

and we need to consider the following sub-cases.

Case (2b)(i). $0<\mathscr{A}<4 \omega^{2}(k)$, with $w(k)>0$ then $a=\hat{a}^{2}>0, b=\hat{b}^{2}>0$. Hence, equation 4.3. becomes

$$
f_{Y}^{2}=\left(f^{2}-\hat{a}^{2}\right)\left(f^{2}-\hat{b}^{2}\right)
$$

In this case the solution of equation 5.23 is

$$
\begin{aligned}
f_{2 b i}(Y)=\hat{b} \operatorname{sn}\left(\hat{a} Y, \frac{\hat{b}^{2}}{\hat{a}^{2}}\right)=\sqrt{2 \omega(k)-\sqrt{4 \omega^{2}(k)-\mathscr{A}}} \\
\times \operatorname{sn}\left(\sqrt{2 \omega(k)+\sqrt{4 \omega^{2}(k)-\mathscr{A}}} Y, \frac{2 \omega(k)-\sqrt{4 \omega^{2}(k)-\mathscr{A}}}{2 \omega(k)+\sqrt{4 \omega^{2}(k)-\mathscr{A}}}\right)
\end{aligned}
$$

For $k=1, \omega=1$ then $\omega(k)=\frac{5}{8}$. If we let $\mathscr{A}=1$ this gives $a=2, b=\frac{1}{2}$, and we thus have

$$
f_{2 b i}(Y)=\frac{\sqrt{2}}{2} \operatorname{sn}\left(\sqrt{2} Y, \frac{1}{4}\right)
$$

see Fig. 11 (blue). 

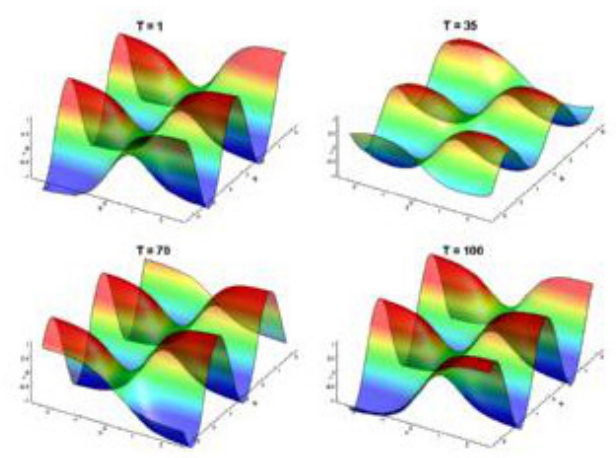

FIG. 10: Time series tanh-solution of cubic nonlinear Schrödinger equation, for the same parameters as that in Fig 9.

Case (2b)(ii). $\mathscr{A}<0<4 \omega^{2}(k)$, with $w(k)>0$ then $a=\hat{a}^{2}>0, b=-\hat{b}^{2}<0$. Hence, equation 4.3. becomes

$$
f_{Y}^{2}=\left(f^{2}-\hat{a}^{2}\right)\left(f^{2}+\hat{b}^{2}\right)
$$

Here the solution of equation 5.26 is

$$
\begin{aligned}
f_{2 b i i}(Y)=\hat{a} \mathrm{nc}\left(\sqrt{\hat{a}^{2}+\hat{b}^{2}} Y, \frac{\hat{b}^{2}}{\hat{a}^{2}+\hat{b}^{2}}\right)=\sqrt{2 \omega(k)+\sqrt{4 \omega^{2}(k)-\mathscr{A}}} \\
\\
\times \mathrm{nc}\left(2 \sqrt{\omega(k)} Y, \frac{2 \omega(k)-\sqrt{4 \omega^{2}(k)-\mathscr{A}}}{4 \omega(k)}\right)
\end{aligned}
$$

For $k=1, \omega=1$ then $\omega(k)=\frac{5}{8}$. Letting $\mathscr{A}=-\frac{11}{16}$ gives $a=\frac{11}{4}, b=-\frac{1}{4}$, and we obtain

$$
f_{2 b i i}(Y)=\frac{\sqrt{11}}{2} \operatorname{nc}\left(\frac{\sqrt{15}}{2} Y, \frac{1}{15}\right)
$$

see Fig. 11 (red).

Case (2b)(iii). $0<\mathscr{A}<4 \omega^{2}(k)$, with $w(k)<0$ then $a=-\hat{a}^{2}<0, b=-\hat{b}^{2}<0$. Hence, equation 4.3. becomes

$$
f_{Y}^{2}=\left(f^{2}+\hat{a}^{2}\right)\left(f^{2}+\hat{b}^{2}\right)
$$

The solution of equation 5.29 is now given by

$$
\begin{aligned}
f_{2 b i i i}(Y)=\hat{a} \operatorname{sc}(\hat{b} Y & \left.\frac{\hat{b}^{2}-\hat{a}^{2}}{\hat{b}^{2}}\right)=\sqrt{2 \omega(k)+\sqrt{4 \omega^{2}(k)-\mathscr{A}}} \\
& \times \operatorname{sc}\left(\sqrt{2 \omega(k)-\sqrt{4 \omega^{2}(k)-\mathscr{A}}} Y, \frac{2 \sqrt{4 \omega^{2}(k)-\mathscr{A}}}{2 \omega(k)-\sqrt{4 \omega^{2}(k)-\mathscr{A}}}\right)
\end{aligned}
$$




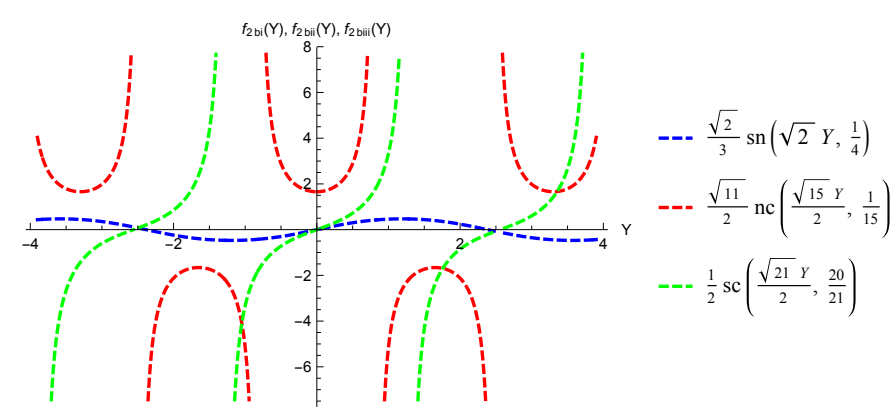

FIG. 11: Solutions when $\mathscr{A}<4 \omega^{2}(k)$, for $k=1, \omega=1, \mathscr{A}=1$ (blue); $k=1, \omega=1, \mathscr{A}=-\frac{11}{16}$ (red); and $k=1, \omega=-1$, $\mathscr{A}=\frac{21}{16}$ (green).

For $k=1, \omega=-1$ then $\omega(k)=-\frac{11}{8}$. Now if $\mathscr{A}=\frac{21}{16}$ gives $a=-\frac{1}{4}, b=-\frac{21}{4}$, and we get

$$
f_{2 b i i i}(Y)=\frac{1}{2} \operatorname{sc}\left(\frac{\sqrt{21}}{2} Y, \frac{20}{21}\right)
$$

see Fig. 11 (green).

\section{WAVE GROUPS GENERATED BY WIND}

Yuen \& Lake [29] proposed that a nonlinear wind-driven wave field may be characterized, to a first approximation, by a single nonlinear wave train. They based this proposal on experimental data obtained earlier for nonlinear deepwater wave trains without wind forcing. Using their laboratory experimental results they argued that the wind-wave interaction transports energy predominantly at a single speed corresponding to the group velocity, which is based on the dominant frequency corrected by wind-induced drift.

Based on their findings, they developed a model for the evolution of a nonlinear wave train in the absence of wind (which they argued is a satisfactory model) through the nonlinear Schrödinger equation

$$
i\left(\frac{\partial \psi}{\partial t}+\frac{\omega_{0}}{2 k_{0}} \frac{\partial \psi}{\partial x}\right)-\frac{\omega_{0}}{8 k_{0}^{2}} \frac{\partial^{2} \psi}{\partial x^{2}}-\frac{1}{2} \omega_{0} \varepsilon^{2}|\psi|^{2} \psi=0
$$

where $\psi$ is the complex wave envelope, $\omega_{0}$ and $k_{0}$ are the carrier-wave frequency and wavenumber, and $\varepsilon=k_{0} a_{0}$ is the inital steepness of the wave train. Similar to our analysis outlined in this paper, they related the free surface $\eta(x, t)$ to the wave envelope by the expression

$$
\eta(x, t)=\operatorname{Re}\left\{\frac{\varepsilon}{k_{0}} \psi e^{i\left(k_{0} x-\omega_{0} t\right)}\right\} .
$$

Yuen \& Lake remarked that in a frame of reference moving with the group velocity $c_{g}=\omega / 2 k_{0}$ the variation of $\psi$ with $x$ and $t$ are of order $\varepsilon k_{0}$ and $\varepsilon^{2} \omega_{0}$, respectively, and is therefore slow compared to the oscillations of the dominant wave, characterized by $k_{0}$ and $\omega_{0}$.

Based on recent studies, e.g. [30, 31, and our analysis here, it is very unlikely that Yuen \& Lake's model can adequately (if at all) represent nonlinear surface waves induced by wind, particularly in three dimensions.

A more consistent model, based on relatively a recent study by Leblanc [32, for three-dimensional deep water waves induced by wind forcing is to adopt the following two-dimensioanl version of nonlinear Schrödinger equation (cf. [33])

$$
2 i \frac{\partial A}{\partial \tau}+\mathscr{C}_{1} \frac{\partial^{2} A}{\partial \xi^{2}}+\mathscr{C}_{2} \frac{\partial^{2} A}{\partial \zeta^{2}}-\mathscr{C}_{3}|\psi|^{2} \psi=\omega(\alpha+i \beta) A
$$

where $\tau, \xi, \zeta$ are slow variables

$$
\xi=\varepsilon\left(x-c_{g} t\right), \quad \zeta=\varepsilon z, \quad \tau=\varepsilon^{2} t,
$$

and $c_{g}=\partial \omega / \partial k$ is the group velocity. The coefficients $\mathscr{C}_{i}, i=1,2,3$ are given by

$$
\mathscr{C}_{1}=\frac{\partial c_{g}}{\partial k}, \quad \mathscr{C}_{2}=C_{g} k^{-1} \quad \text { and } \quad \mathscr{C}_{3}=4 k^{4} \omega^{-1} .
$$



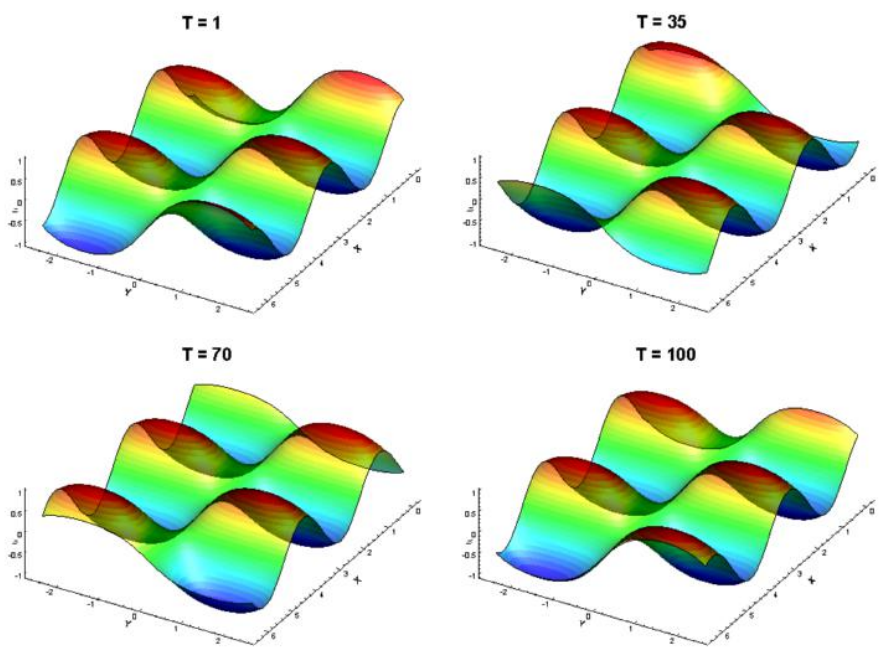

FIG. 12: Time series sn-solution of cubic nonlinear Schrödinger equation, for the same parameters as that in Fig 11.

On the right-hand side of equation $\sqrt[6.2]{ }, \alpha$ and $\beta$ are the interfacial impedance, related to normal and tangential stresses at the air-sea interface [34]; $\beta$ is commonly known as the energy-transfer parameter.

A solution of equation 6.2 may be sought in the form

$$
A(\xi, \zeta, \tau)=\left(\omega / 2 k^{2}\right) \psi(X, Z, T) e^{-i \alpha T / 2}
$$

where $T=\omega \tau, X=k \xi$ and $Z=k \zeta$. An exact homogeneous solution of 6.2 maybe obtained by writing $\psi_{s}(T)=$ $\mathscr{R}_{s} e^{i \Theta_{s}} 32$ where

$$
\mathscr{R}_{s}(T)=\mathscr{R}_{0} e^{\beta T / 2}, \quad \Theta_{s}(T)=\Theta_{0}-\mathscr{R}_{s}^{2} / 2 \beta
$$

where the suffix zero indicates the initial state. The solution corresponding to a spatially uniform plane Stokes wave is then obtained by letting $\beta \rightarrow 0$ and in this limit $\mathscr{R}_{s} \rightarrow \mathscr{R}_{0} e^{i\left(\Theta_{0}-\mathscr{R}_{0}^{2} T / 2\right)}$. On the other hand $\psi_{s}$ for an inhomogeneous solution is of the form $\psi_{s}(T)=e^{i K X+L Z}$ provided $K^{2}=2 L^{2}$.

Following Leblanc 32 , we seek a perturbation to the Stokes solution by writing

$$
\psi=\mathscr{R}_{s}(1+\mu) e^{i\left(\Theta_{s}+\varphi\right)}
$$

and expressing $\mu$ and $\varphi$ in the form

$$
[\mu, \varphi]=[\Lambda(T), \Phi(T)] \cos (K X+L Z)
$$



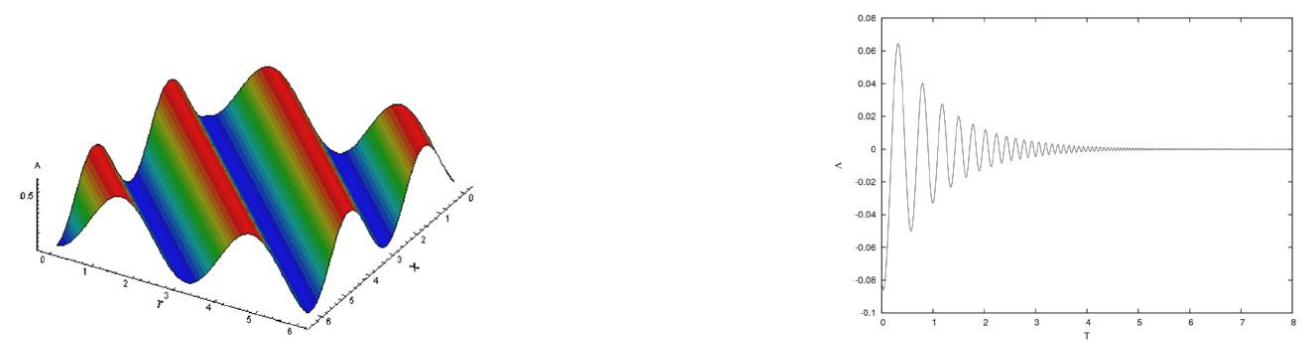

FIG. 13: (Left) Formation of a surface wave with wind forcing, calculated from $\beta$ by Miles' 34 formula, obtained from sn-solution of cubic nonlinear Schrödinger equation given in case (2b)(i) above. (Right) Variation of $\Lambda$ with $T$ for $\beta<0$.

where $\Lambda$ satisfies the following ordinary differential equation

$$
\Lambda^{\prime \prime}+\gamma\left(\gamma-\mathscr{R}_{s}^{2}\right) \Lambda=0, \quad \gamma=\frac{1}{8}\left(K^{2}-2 L^{2}\right) ; \quad()^{\prime} \equiv \mathrm{d} / \mathrm{d} T
$$

As in the analysis of Leblanc 32 for two-dimensional waves, we see that in the absence of wind forcing $(\beta=0)$ the solution grows exponentially provided $0<\gamma<\mathscr{R}_{0}^{2}$. This represents the three-dimensional Benjamin-Feir instability and is in direct contradiction with Yuen \& Lake [29. However, when wind forcing is present $(\beta \neq 0)$, it is easy to show the differential equation (6.4) admits two linearly independent solutions in terms of modified Bessel function $I_{ \pm \nu}(f)$ of complex order $\nu=2 i \gamma \beta^{-1}$ where $f(T)=2 \gamma^{\frac{1}{2}} \mathscr{R}_{s} \beta^{-1}$. Thus we see that the decaying $(\beta<0)$ solution of equation (6.4) oscillates in time and eventually damps due to viscous dissipation. On the other hand, the nondissipative solution $(\beta>0)$ either decays if $\gamma<0$ or grows superexponentially [32] when $\gamma>0$. In the latter case the asymptotic behaviour of equation 6.4 is given by 32 ]

$$
\Lambda(T) \sim \frac{e^{f(T)}}{\sqrt{2 \pi f(T)}} \quad \text { as } T \rightarrow \infty .
$$

This results seems to suggest the three-dimensional waves for which $\beta>0$ suffer superharmonic instability initially but they are then suppressed by subharmonic instability. However, as yet no experimental evidence has been reported to support or reject this conjecture.

\section{CONCLUDING REMARKS}

In this paper we have reviewed several types of three-dimensional waves on deep-water. We have identified that three dimensionality of surface waves are essentially of three kinds namely those of oblique, forced and spontaneous 

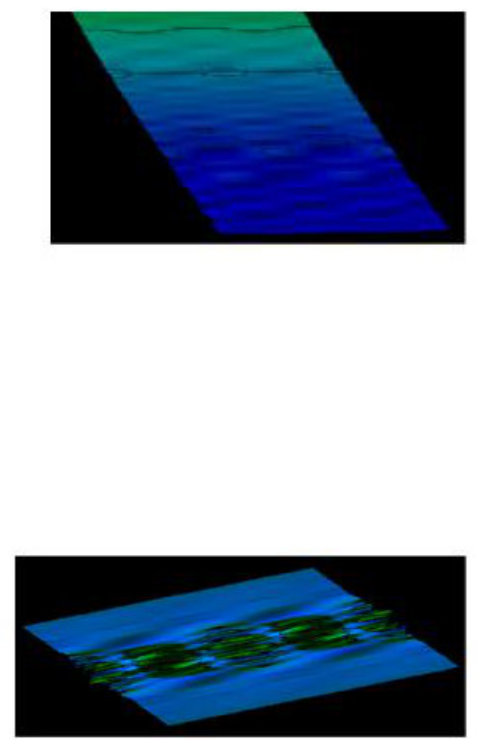

FIG. 14: (Top) Formation of wave groups which gradually grow in time. (Bottom) Formation of wave groups with initial growth and subsequent decay in time.

type. Although perturbation techiques can be used to describe these waves, we have shown an alternative formulation, through cubic nonlinear Schrödinger (NLS) equation, which in some cases may be superior. It is shown that, when adopting the alternative approach the solution of NLS equation must be carefully classified since this equation has overabundance of solutions and it is often difficult to decide which, if any, have physcial significance. Here, we have obtained various periodic solutions of the NLS equation using Weierstrass elliptic $\wp$ functions. It is shown the classification of solutions depends on the boundary conditions, wavenumber and frequency. We have demonstrated that in certain cases the solutions are of solitary type, or simply periodic, while in other cases it is shown that solutions can be expressed in terms of Jacobi elliptic functions. For the formation of a group of waves, analytical solution of forced (or inhomogeneous) NLS equation, which arises from wind forcing, is found whose that clearly shows group of waves can form on the surface of deep water similar to those that are commonly observed in the ocean. In this case the depencency on the energy-transfer parameter, from wind to waves, is clearly a very influential factor, making either the groups of wave to grow initially and eventually dissipate or simply decay or grow in time.

\section{Appendix A: Second and third order interaction coefficients}

Adopting the short-hand notation $T\left(k, k_{1}, k_{2}, k_{3}\right)=T_{0,1,2,3}$ we have

$$
\begin{aligned}
T_{0,1,2,3}= & -\frac{2 \mathscr{V}_{3,3-1,1}^{(-)} \mathscr{V}_{0,2,0-2}^{(-)}}{\omega_{1-3}-\omega_{3}+\omega_{1}}-\frac{2 \mathscr{V}_{2,0,2-0}^{(-)} \mathscr{V}_{1,1-3,3}^{(-)}}{\omega_{1-3}-\omega_{1}+\omega_{3}}-\frac{2 \mathscr{V}_{2,2-1,1}^{(-)} \mathscr{V}_{0,3,0-3}^{(-)}}{\omega_{1-2}-\omega_{2}+\omega_{1}} \\
& -\frac{2 \mathscr{V}_{3,0,3-0}^{(-)} \mathscr{V}_{1,1-2,2}^{(-)}}{\omega_{1-2}-\omega_{1}+\omega_{2}}-\frac{2 \mathscr{V}_{0+1,0,1}^{(-)} \mathscr{V}_{2+3,2,3}^{(-)}}{\omega_{2+3}-\omega_{2}-\omega_{3}}-\frac{2 \mathscr{V}_{-2-3,2,3}^{(+)} \mathscr{V}_{0,1,-0-1}^{(+)}}{\omega_{2+3}+\omega_{2}+\omega_{3}} \\
& +\mathscr{W}_{0,1,2,3}
\end{aligned}
$$


Here, the second order interaction coefficients $\mathscr{V}_{0,1,2}^{( \pm)}$are given by

$\mathscr{V}_{0,1,2}^{( \pm)}=\frac{1}{8 \sqrt{2} \pi}\left\{\left(\boldsymbol{k}_{0} \cdot \boldsymbol{k}_{1} \pm k_{0} k_{1}\right)\left(\frac{\omega_{0} \omega_{1}}{\omega_{2}} \frac{k_{2}}{k_{0} k_{1}}\right)^{\frac{1}{2}}+\left(\boldsymbol{k}_{0} \cdot \boldsymbol{k}_{2} \pm k_{0} k_{2}\right)\left(\frac{\omega_{0} \omega_{2}}{\omega_{1}} \frac{k_{1}}{k_{0} k_{2}}\right)^{\frac{1}{2}}+\left(\boldsymbol{k}_{1} \cdot \boldsymbol{k}_{2} \pm k_{1} k_{2}\right)\left(\frac{\omega_{1} \omega_{2}}{\omega_{0}} \frac{k_{0}}{k_{1} k_{2}}\right)^{\frac{1}{2}}\right\}$

where $k_{i}=\left|\boldsymbol{k}_{i}\right|$ and $\omega_{i}=\omega\left(k_{i}\right)$.

Similarly, writing the short-hand notation $\mathscr{W}_{0,1,2,3}=\mathscr{W}\left(\boldsymbol{k}, \boldsymbol{k}_{1}, \boldsymbol{k}_{2}, \boldsymbol{k}_{3}\right)$ we have for the third order interaction coefficients

$$
\mathscr{W}_{0,1,2,3}=\mathscr{U}_{-0,-1,2,3}+\mathscr{U}_{2,3,-0,-1}-\mathscr{U}_{2,-1,-0,3}-\mathscr{U}_{-0,2,-1,3}-\mathscr{U}_{-0,3,2,-1}-\mathscr{U}_{3,-1,2,-0}
$$

where

$$
\mathscr{U}_{0,1,2,3}=\frac{1}{64 \pi^{2}}\left(\frac{\omega_{0} \omega_{1}}{\omega_{2} \omega_{3}} k_{0} k_{1} k_{2} k_{3}\right)^{\frac{1}{2}}\left\{2\left(k_{0}+k_{1}\right)-k_{1+3}-k_{1+2}-k_{0+3}-k_{0+2}\right\}
$$

with $k_{i \pm j}=\left|\boldsymbol{k}_{i} \pm \boldsymbol{k}_{j}\right|$ and $\omega_{i \pm j}=\omega\left(k_{i \pm j}\right)$.

\section{References}

[1] G.G. Stokes, On the theory of oscillatory waves. Trans. Camb. Phil. Soc. 8 (1847) 441.

[2] M.J. Lighthill, Contributions to the theory of waves in nonlinear dispersive systems. J. Inst. Math. Appl. 1 (1965) 269.

[3] V.E. Zakharov, Stability of periodic waves of finite amplitude on a surface of deep fluid. J. Appl. Mech. Tech. Phys. 9 (1968) 190.

[4] T.B. Benjamin \& J.E. Feir, The disintegration of wave trains on deep water. Part 1. Theory. J. Fluid Mech. 27 (1967) 417.

[5] T.B. Benjamin \& J.E. Feir, The disintegration of wave trains on deep water. Part 2. Experiments. (Unpublished) (1967).

[6] J.E. FeIR, Some Results From Wave Pulse Experiments. Proc. Roy. Soc. A299 (1967) 54.

[7] D.L. Ross \& S.G. SajJadi, On the disintegration of oblique waves on deep water. Adv. Appl. Fluid Mech. 3 (2008) 23.

[8] S.G. SajJadi, A note on Benjamin-Feir instability for water waves. Adv. Appl. Fluid Mech. (2008) In press.

[9] W.G. Penney \& A.J. Price, Finite periodic stationary gravity waves in a perfect liquid. Phil. Trans. R. Soc. Lond. A224 (1952) 254.

[10] M.S. Longuet-Higgins, The instabilities of gravity waves of finite amplitude in deep water. I. Superharmonics. Proc. Roy. Soc. A360 (1978) 471.

[11] M.S. Longuet-Higgins, The instabilities of gravity waves of finite amplitude in deep water. II. Subharmonics. Proc. Roy. Soc. A360 (1978) 489.

[12] T.B. Benjamin, Instability of periodic wavetrains in nonlinear dispersive systems. Proc. Roy. Soc. A299 (1967) 59.

[13] B.M. LAKE \& H.C. Yuen, A note on some nonlinear water wave experiments and the comparison of data with theory. J. Fluid Mech. 83 (1977) 75.

[14] M-Y. Su, M. Bergin, P. Marler \& R. Myrick, Experiments on nonlinear instabilities and evolution of steep gravitywave trains. J. Fluid Mech. 124 (1982) 45.

[15] P.G. Saffman \& H.C. Yuen, A new type of three-dimensional deep-water wave of permanent form. J. Fluid Mech. 101 (1980) 797.

[16] R.A. Fuchs, On the theory of short-crested oscillatory grvity waves. Natl. Bur. Standards Circ. 521 (1951) 187.

[17] J.C. Chappelear, On the description of short crested waves, Army Corps of Engrs. Tech. Memo no. 125 (1961).

[18] L. Schwartz \& A.K. Whitney, A semi-analytic solution for nonlinear standing waves in deep water. J. Fluid Mech. 107 (1981) 147.

[19] P.G. Saffman \& H.C. Yuen, Bifurcation and symmetry breaking in nonlinear dispersive waves. Phys. Rev. Let. 4 (1980) 1097.

[20] D.I. Meiron, P.G. Saffman \& H.C. Yuen, Calculation of steady three-dimensional deep-water waves. J. Fluid Mech. 124 (1982) 109.

[21] M-Y. Su, Three-dimensional deep-water waves. Part 1. Experimental measurement of skew and symmetric wave patterns. J. Fluid Mech. 124 (1982) 73.

[22] J.W. Mclean, D.U. Martin, P.G. Saffman \& H.C. Yuen, Three-dimensional instability of finite amplitude gravity waves. Phys. Rev. Let. 46 (1981) 817.

[23] D.R. Crwaford, P.G. Saffman \& H.C. Yuen, Evolution of a random inhomogeneous field of nonlinear deep-water gravity waves. Wave Motion 2 (1980) 1.

[24] P.G. Saffman \& H.C. Yuen, Stability of a plane soliton to a two-dimensional perturbations. Phys. Fluids 21 (1978) 1450 . 
[25] A.J. Roberts \& D.H. Peregrine, Notes on long-crested water waves. (unpublished) (1983).

[26] K. Weierstrass, Mathematische Werke, vol. V. Johnson, New York (1915).

[27] E.T. Whittaker \& G.N. Watson, A Course of Modern Analysis. Cambridge Univesity Press, Cambridge (1927).

[28] M. Abramowitz \& I.A. Stegun, Handbook of Mathematical Functions: With Formulas, Graphs, and Mathematical Tables. Courier Dover Publications (1972).

[29] H.C. Yuen \& B.M. Lake, Nonlinear dynamics of deep-water gravity waves. Advances in Applied Mech. 22 (1982) 67.

[30] S.G. SajJadi, J.C.R. Hunt \& F. Drullion, Asymptotic multi-layer analysis of wind over unsteady monochromatic surface waves. J. Eng. Maths, 84 (2014) 73.

[31] S.G. Sajuadi, J.C.R. Hunt \& F. Drullion, Dynamics of critical layer in turbulent shear flows over unsteady water waves. J. Fluid Mech. (Submitted) (2014).

[32] S. Leblanc, Amplification of nonlinear surface waves by wind. Phys. Fluids 19 (2007) 101.

[33] S.G, SajJadi \& H. Khanal, Interaction of Tollmien-Schlichting waves in the air with the sea surface. Adv. Appl. Fluid Mech. 5 (2008) 69.

[34] J.W. MiLEs, On the generation of surface waves by shear flows. J. Fluid Mech. 3 (1957) 185. 